\title{
Inhibitory Effect of Standardized Curcuma xanthorrhiza Supercritical Extract on LPS-Induced Periodontitis in Rats
}

\author{
Kyo Eun Kook ${ }^{1}$, Changhee Kim ${ }^{1}$, Wonku Kang ${ }^{2}$, and Jae-Kwan Hwang ${ }^{1 *}$ \\ ${ }^{1}$ Department of Biotechnology, College of Life Science and Biotechnology, Yonsei University, Seoul 03722, Republic of Korea \\ ${ }^{2}$ College of Pharmacy, Chung-Ang University, Seoul 06974, Republic of Korea
}

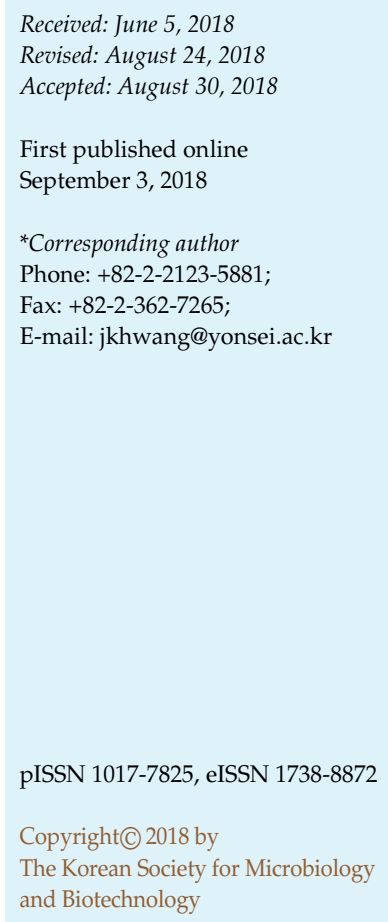

Periodontitis, which is a severe inflammatory disease caused by endotoxins secreted from oral pathogens, destructs gingival tissue and alveolar bone. Curcuma xanthorrhiza, commonly called Java turmeric, has been shown to possess anti-bacterial and anti-inflammatory activities. The present study evaluated the inhibitory effect of C. xanthorrhiza supercritical extract (CXS) standardized with xanthorrhizol on lipopolysaccharide (LPS)-induced periodontitis in an animal model. LPS was topically injected into the periodontium of Sprague-Dawley rats to induce periodontitis and CXS (30 and $100 \mathrm{mg} \cdot \mathrm{kg}^{-1} \cdot \mathrm{day}^{-1}$ ) was orally administered after day 12 . Histologically, CXS inhibited the collapse of gingival tissue by preventing cell infiltration. CXS significantly downregulated the expression of matrix metalloproteases (MMPs) and inflammation-related biomarkers, such as nuclear factor-kappa B (NF- $\mathrm{kB})$ and interleukin-1 beta (IL-1 $\beta$ ) in gingival tissue. CXS also improved bone remodeling by downregulating osteoclastic transcription factors, such as nuclear factor of activated T-cells c1 (NFATc1), tartrate-resistant acid phosphatase (TRAP), and cathepsin K. In addition, CXS upregulated osteoblast differentiation-related markers, alkaline phosphate (ALP) and collagen type I alpha (COLA1). Thus, CXS can ameliorate periodontitis by inhibiting inflammation and improving bone remodeling.

Keywords: Curcuma xanthorrhiza, osteoblastogenesis, osteoclastogenesis, periodontitis, periodontal inflammation

\section{Introduction}

Periodontal disease is generally classified into gingivitis and periodontitis according to the degree of inflammation [1]. Gingivitis is a temporary inflammation in gingival tissue on which oral pathogens infect, but it is easily recovered from by scaling or brushing the teeth [2]. Meanwhile, periodontitis is a chronic disease associated with severe inflammation in both gingival tissue and alveolar bone [3]. Periodontitis weakens the ability to support teeth, consequently limiting the mastication of foods and reducing quality of life. In addition, periodontitis is closely related to systemic diseases, such as cardiovascular disease, hypertension, cancer, and dementia [4-6]. Therefore, the prevention or treatment of periodontitis is important in improving oral health and ward off systemic diseases.
Gram-positive organisms, such as Streptococcus mutans (S. mutans) form a plaque/biofilm on the surface of teeth to provide circumstances for the growth of Porphyromonas gingivalis ( $P$. gingivalis) or a representative oral pathogen to induce periodontitis [7]. P. gingivalis living in plaque/biofilm secretes lipopolysaccharide (LPS), which is an endotoxin that induces inflammation, subsequently collapsing the periodontal ligament, which is a tissue responsible for connecting between teeth and gingiva [8]. The resulting deterioration leads to the resorption of alveolar bone and the destruction of gingival tissues [9].

LPS binds to toll-like receptors (TLRs) of gingival fibroblasts, activating nuclear factor kappa-B (NF-кB) [10]. Activated NF- $\kappa B$ upregulates matrix metalloproteinases (MMPs) [11] and interleukin-1 beta (IL-1 $\beta$ ), which is a major cytokine for periodontitis [1]. MMP-2 and MMP-8 are major MMP 
subfamilies that degrade protein matrix components of gingival tissues and periodontal ligaments. MMP-2 is a collagen-degrading enzyme that is strongly expressed when inflammation occurs in the periodontal tissue [12]. In addition, MMP-8 is a major collagenase and is commonly observed in saliva when periodontal disease occurs [13].

In the condition of continuous inflammation, which is induced by LPS, gingival fibroblasts or osteoblasts oversecrete the receptor activator of nuclear factor kappa-B ligand (RANKL), which is an activator of osteoclastogenesis or a process of osteoclast formation causing bone resorption [14]. When secreted RANKL attaches to the receptor activator of nuclear factor kappa-B (RANK), osteoclastogenesis starts with the upregulation of the major osteoclast-specific genes, such as nuclear factor of activated T-cell c1 (NFATc1), tartrate resistant acid phosphatase (TRAP), and cathepsin $\mathrm{K}$ [15]. However, since osteoprotegerin (OPG) is a decoy receptor of RANKL, increasing the expression of OPG inhibits or delays osteoclastogenesis-related molecular mechanisms [9]. Therefore, the ratio between OPG and RANKL denotes bone homeostasis.

Curcuma xanthorrhiza (C. xanthorrhiza) Roxb., commonly called Java turmeric, belongs to the Zingiberaceae family. C. xanthorrhiza has been shown to possess anti-bacterial [16], anti-fungal [17], anti-halitosis [18], and anti-inflammatory activities [19]. C. xanthorrhiza contains several phytochemicals, including $\alpha$-pinene, camphor, curcumin, and xanthorrhizol [20]. In particular, xanthorrhizol exists in greater abundance than other compounds in C. xanthorrhiza, conferring antimicrobial, anti-fungal, anti-inflammatory, and anti-cancer activities [21]. The current study investigated whether C. xanthorrhiza supercritical extract (CXS) standardized with xanthorrhizol attenuated LPS-induced periodontitis in rats by identifying the levels of inflammatory factors and bone homeostasis-related markers.

\section{Materials and Methods}

\section{Chemical Reagents}

Escherichia coli LPS and protease inhibitor cocktails were supplied by Sigma-Aldrich (USA). Primary antibodies against NF- $\kappa B$, IL$1 \beta$, MMP-2, MMP-8, TRAP, NFATc1, cathepsin K, c-Fos, phospho (p)-extracellular signal-regulated kinase (ERK), p-c-Jun N-terminal kinase (JNK), p-p38, p-c-Jun, c-Jun, ERK, JNK, p38, alkaline phosphatase (ALP), collagen type 1 alpha 1 (COLA1), OPG, and RANKL were obtained from Santa Cruz Biotechnology Inc. (USA). $\alpha$-Tubulin was obtained from Cell Signaling (USA). Horseradish peroxidase-conjugated IgG secondary antibody was purchased from Bethyl Laboratories, Inc. (USA).

\section{Preparation of Standardized CXS}

The rhizomes of C. xanthorrhiza were supplied by Nutribiotech Co. (Korea) and extracted using a supercritical $\mathrm{CO}_{2}$ fluid extraction system (SCFE-P400, Ilshin Autoclave Co., Ltd., Korea). The ground rhizomes were placed in a high-pressure vessel and the oven at $50^{\circ} \mathrm{C} . \mathrm{CO}_{2}$ was pressurized using a high-pressure pump and then charged into the vessel at the rate of $3.21 / \mathrm{min}$ to maintain the pressure at $40 \mathrm{MPa}$. The yield of CXS was $8.0 \%(\mathrm{w} / \mathrm{w})$. CXS was standardized to contain $30.0 \%(\mathrm{w} / \mathrm{w})$ of xanthorrhizol as an active compound [17].

\section{Animal Experiment}

Forty male, 9-week-old Sprague-Dawley rats with initial mean weight of 280-300 g were bred in a controlled environment $(24 \pm$ $2^{\circ} \mathrm{C}$ temperature and $55 \pm 5 \%$ relative humidity with a $12 \mathrm{~h}$ lightdark cycle) at the College of Pharmacy, Chung-Ang University (Korea). After a week of circulation, the forty rats were randomly assigned into four groups: (i) Control (corn oil); (ii) LPS (LPS injection); (iii) LPS+CXS-L (LPS injection+CXS $30 \mathrm{mg} \cdot \mathrm{kg}^{-1} \cdot \mathrm{day}^{-1}$ ), and (iv) LPS+CXS-H (LPS injection+CXS $100 \mathrm{mg} \cdot \mathrm{kg}^{-1} \cdot \mathrm{day}^{-1}$ ). All the groups except control, were injected with $1 \mathrm{mg} / \mathrm{ml}$ of LPS bilaterally between the first and second mandible molars every two days under anesthesia with isoflurane (Hana Pharm. Co. Ltd., Korea) before sacrifice. Oral administration of CXS at the dose of 30 and $100 \mathrm{mg} \cdot \mathrm{kg}^{-1} \cdot \mathrm{day}^{-1}$ was started after six LPS injections and daily conducted for eight days, followed by sacrifice. Periodontal tissues were isolated from each rat. Gingival tissues and alveolar bones were separated from isolated periodontal tissues. The animal experiment was according to the protocol as reviewed and approved by the Institutional Animal Care and Use Committee (IACUC) of the Chung-Ang University Laboratory Animal Research Center (Permit No.: 2017-00024).

\section{Reverse Transcription-Polymerase Chain Reaction (RT-PCR)}

Total RNA was isolated from the gingival tissue and alveolar bone using Trizol reagent (Takara, Japan). The concentration of mRNA was quantified using NanoDrop Lite (Thermo Fisher Scientific Inc., USA). The quantified mRNA was denatured at $70^{\circ} \mathrm{C}$ for $5 \mathrm{~min}$ and added in reverse to transcriptase premix (Elpis Biotech, Korea) to synthesize mRNA into cDNA. The reaction of reverse transcription (RT) was started at $42^{\circ} \mathrm{C}$ for $55 \mathrm{~min}$ and then terminated at $70^{\circ} \mathrm{C}$ for $15 \mathrm{~min}$. The synthesized cDNA was amplified with specific primer pairs (Bioneer, Korea) (Table 1) for 30-35 cycles as follows: denaturation at $94^{\circ} \mathrm{C}$ for $30 \mathrm{sec}$, annealing for $1 \mathrm{~min}$ at each specific temperature dependent on primer, extension at $72^{\circ} \mathrm{C}$ for $1 \mathrm{~min}$. Final extension was performed at $72^{\circ} \mathrm{C}$ for $5 \mathrm{~min}$. The products of PCR were stained with Loading STAR (Dynbio, Korea), separated using 1.5\% agarose gel electrophoresis, and detected with the G:BOX EF imaging system (Syngene) and the Genesys software program. The intensity of target gene expression was measured using ImageJ software (National Institutes of Health, USA). 
Table 1. Primer sequences used in RT-PCR analysis.

\begin{tabular}{|c|c|c|}
\hline Gene & Direction & Sequence $\left(5^{\prime}-3^{\prime}\right)$ \\
\hline \multirow[t]{2}{*}{ IL-1 $\beta$} & Forward & AGC ACC TTC TTT TCC TTC ATC TTT G \\
\hline & Reverse & TTC TAT CTT GTT GAA GAC AAA CCG C \\
\hline \multirow[t]{2}{*}{ NF-кB } & Forward & CTC GAC CTC CAC CGG ATC TT \\
\hline & Reverse & CTG TTT AGG CTG TTC CAC AAT CAC \\
\hline \multirow[t]{2}{*}{ MMP-2 } & Forward & GTC TGA AGA GTG TGA AGT TTG GAA G \\
\hline & Reverse & GCT GTA ACC CAC AAA AGA TCA TTC A \\
\hline \multirow[t]{2}{*}{ MMP-8 } & Forward & CAA TTT CAT ATC TCT GTT CTG GCC C \\
\hline & Reverse & CTG CTG GAA AAC TGC ATC AAT TCT A \\
\hline \multirow[t]{2}{*}{ TRAP } & Forward & AGA ATA AAG TCT CAG CGA TCA CC \\
\hline & Reverse & TCA GAG AAC ACA TCC TCA AAG GTC \\
\hline \multirow[t]{2}{*}{ Cathepsin K } & Forward & TTA CAG CAG AGG TGT GTA CTA TGA C \\
\hline & Reverse & TTG AGG AAG GAA TGT GAG AAC AGA T \\
\hline \multirow[t]{2}{*}{ NFATc1 } & Forward & CGT GTT AGC AAT AAC CAG TAT CCA C \\
\hline & Reverse & CTT ACT CAT AAC CAC TTT CGG ATG C \\
\hline \multirow[t]{2}{*}{ OPG } & Forward & TCC CTC TGA AGA TTT GAT TCG AGT T \\
\hline & Reverse & GCT TAG GTA CAA CTA CAG AGG AAC A \\
\hline \multirow[t]{2}{*}{ RANKL } & Forward & AAC CAA GAT GGC TTC TAT TAC CTG T \\
\hline & Reverse & AGA ATT CCC TGA CCA GTT CTT AGT G \\
\hline \multirow[t]{2}{*}{ ALP } & Forward & CTC GGA CAA TGA GAT GCG CC \\
\hline & Reverse & TCA GGT TGT TCC GAT TCA ACT CAT A \\
\hline \multirow[t]{2}{*}{ COLA1 } & Forward & GAA GAC CTA TGT GGG TAT AAG TCC C \\
\hline & Reverse & AGA TGG TTA GGC TCC TTC AAT AGT C \\
\hline \multirow[t]{2}{*}{$\beta$-Actin } & Forward & CGA GTA CAA CCT TCT TGC AGC TC \\
\hline & Reverse & CCA AAT CTT СТC CAT ATC GTC CCA G \\
\hline
\end{tabular}

\section{Western Blotting}

The gingival tissues and alveolar bones were homogenized and proteins were extracted using NP40 lysis solution (Elpis Biotech) containing a protease inhibitor cocktail. The extracted proteins were quantified using the Bradford protein assay (Bio-Rad Laboratories Inc., USA) and $20 \mu$ g of proteins in every group were separated using sodium dodecyl sulfate-polyacrylamide gel electrophoresis (SDS-PAGE). Separated proteins were transferred onto nitrocellulose membranes (Whatman $\mathrm{GmBH}$, Germany). After blocking transferred proteins with 5\% skim milk in Trisbuffered saline containing $0.1 \%$ Tween-20 (TBST) for $1 \mathrm{~h}$ at room temperature, the membranes were washed with TBST for $10 \mathrm{~min}$ three times. The proteins on the membranes were incubated with primary antibodies overnight at $4^{\circ} \mathrm{C}$. The membranes bound with primary antibodies were incubated with secondary antibodies for $2 \mathrm{~h}$ at $4^{\circ} \mathrm{C}$. An enhanced chemiluminescence (ECL) detection system (Amersham Biosciences, UK) was used to develop the blots. Protein bands were detected using the G:BOX EF imaging system (Syngene, UK) and Genesys software. The intensity of protein bands was measured using ImageJ software (National Institutes of Health).

\section{Hematoxylin \& Eosin (H\&E) Staining}

The periodontal tissues were fixed with $10 \%$ formalin buffer solution, embedded in paraffin, cut into $5 \mu \mathrm{m}$ sections, and then mounted on slides. The tissues mounted on slides were stained with H\&E. Cell infiltration and alveolar bone resorption were analyzed using an Eclipse TE2000U Inverted Microscope with twin CCD cameras (magnification, $\times 200$; Nikon, Japan).

\section{Immunohistochemistry}

The paraffin slides were incubated with primary antibodies against MMP-2, MMP-8, TRAP, NFATc1, and ALP at $4^{\circ} \mathrm{C}$ overnight. The slides were then incubated with secondary antibody for $2 \mathrm{~h}$ at $4^{\circ} \mathrm{C}$. The proteins in the stained areas were analyzed using an Eclipse TE2000U Inverted Microscope with twin CCD cameras (magnification, $\times 400$; Nikon). The protein expression levels in the stained areas of the image were quantified using ImageJ software (National Institutes of Health).

\section{Micro-Computed Tomography (CT) Imaging}

For quantitative 3D analysis, each sample was vertically placed on the sample holder of a Skyscan 1076 desktop X-ray micro-CT system (Skyscan, Belgium) under the following conditions: total rotation, $360^{\circ}$; rotation step, $0.5^{\circ}$; pixel size, $18 \mu \mathrm{m}$; voltage, $100 \mathrm{kV}$; current, $100 \mu \mathrm{A}$; and exposure time, 1,475 ms. The bone volume per tissue volume (BV/TV), trabecular thickness (Tb.Th.), trabecular separation (Tb.Sp.), and bone mineral density (BMD) on alveolar bone of the first molar were measured by reconstructing the $3 \mathrm{D}$ trabecular structure from the scanned images by NRecon (Skyscan) and CTAn (Skyscan). In addition, the alveolar bone loss was evaluated by analyzing the distance between the alveolar bone crest $(\mathrm{ABC})$ and the cementoenamel junction (CEJ). The distance was quantified using ImageJ software (National Institutes of Health).

\section{Statistical Analysis}

Statistical analysis was performed using the Statistical Package for the Social Sciences (SPSS) version 24.0 (SPSS Inc., USA). Data are presented as mean \pm standard deviation (SD) and group differences were assessed using one-way Analysis of Variance (ANOVA), followed by Duncan's multiple range test. ${ }^{\#} p<0.05$ and ${ }^{\# \#} p<0.01$ were considered to be statistically significant between control group and LPS group. ${ }^{*} p<0.05$ and ${ }^{* *} p<0.01$ were considered to be statistically significant between LPS group and sample groups.

\section{Results}

\section{CXS Alleviates Periodontitis by Reducing Gingival Inflammation and Alveolar Bone Resorption}

As periodontitis is an inflammatory disease, which occurs on the gingival fibers, sequentially resorbing alveolar bone through osteoclastogenesis [3], the inhibitory effect of CXS 

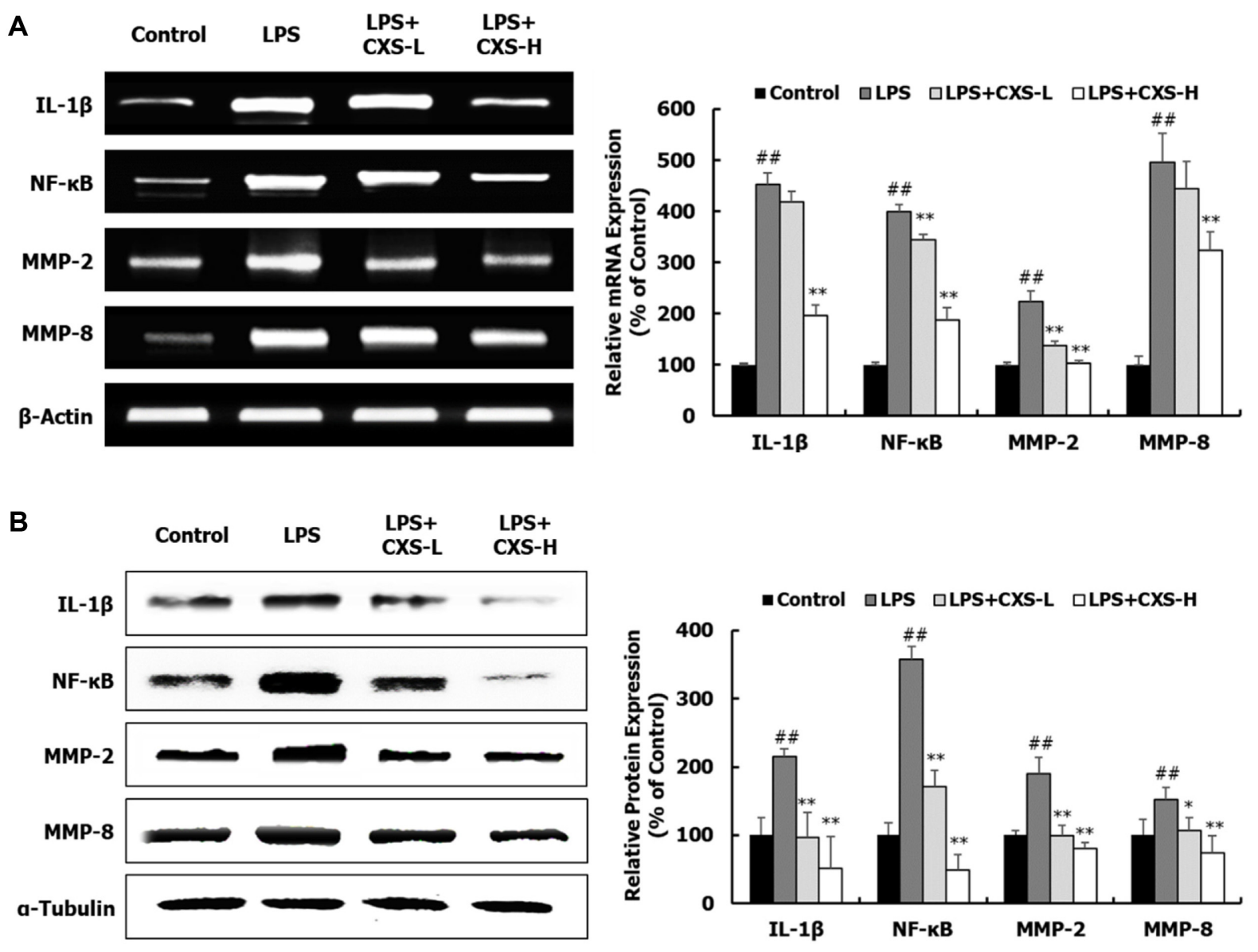

Fig. 1. Inhibitory effect of CXS on inflammation in gingival tissue of LPS-treated rats.

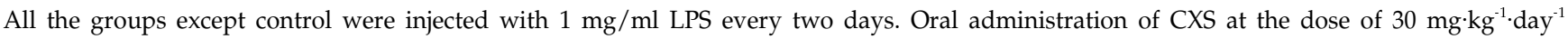
(LPS+CXS-L) and $100 \mathrm{mg} \cdot \mathrm{kg}^{-1} \cdot \mathrm{day}^{-1}$ (LPS+CXS-H) was started after six LPS injections and daily conducted for eight days. (A) mRNA levels of NF$\kappa B$, IL- $\beta$, MMP-2, and MMP-8 were measured using RT-PCR with $\beta$-actin as the housekeeping gene. (B) Protein levels of NF- $\mathrm{B}$, IL- $\beta$, MMP-2, and MMP-8 were measured by western blotting with $\alpha$-tubulin as the housekeeping gene. The relative expression levels were expressed as mean \pm standard deviation (SD, \% control) and group differences were assessed by Duncan's multiple range tests. ${ }^{\#} p<0.01$ (Control group vs. LPS group); ${ }^{*} p<0.05$ and ${ }^{* *} p<0.01$ (LPS group vs. sample groups).

on periodontitis was assessed in terms of anti-inflammation in gingiva tissue and anti-osteoclastogenesis in alveolar bone. The mRNA expression of IL-1 1 , NF-kB, MMP-2, and MMP-8 was decreased by $56.54 \%, 53.19 \%, 54.22 \%$, and $34.92 \%$, respectively, in the CXS-H group, compared to the LPS group (Fig. 1A). In addition, CXS decreased the protein expression of MMPs and gingival inflammation in a concentration-dependent manner, compared to the LPS group (Fig. 1B). To determine whether CXS suppressed LPS-induced osteoclastogenesis, the alveolar bone at the mandible molars was used. The mRNA expression of the bone degradation enzymes, such as TRAP and cathepsin K and the osteoclastic transcription factor, including NFATc1, was decreased in the CXS groups (Fig. 2A). Specifically, the
mRNA expression of TRAP, cathepsin K, and NFATc1 in the CXS-H group was diminished by $69.09 \%, 53.10 \%$, and $54.51 \%$, respectively, compared to the LPS group. As shown in Fig. 2B, these osteoclastogenesis-related markers were also evaluated at the protein level. CXS downregulated the protein expression of TRAP, cathepsin K, and NFATc1, while the expression in the LPS group was significantly increased.

\section{CXS Regulates Osteoclastogenesis by Inactivating MAPKs/AP-1 Signaling Pathway}

The MAPK/AP-1 signaling pathway is a major regulator of osteoclastogenesis for alveolar bone resorption in periodontitis [22]. In the CXS groups, the protein expression 

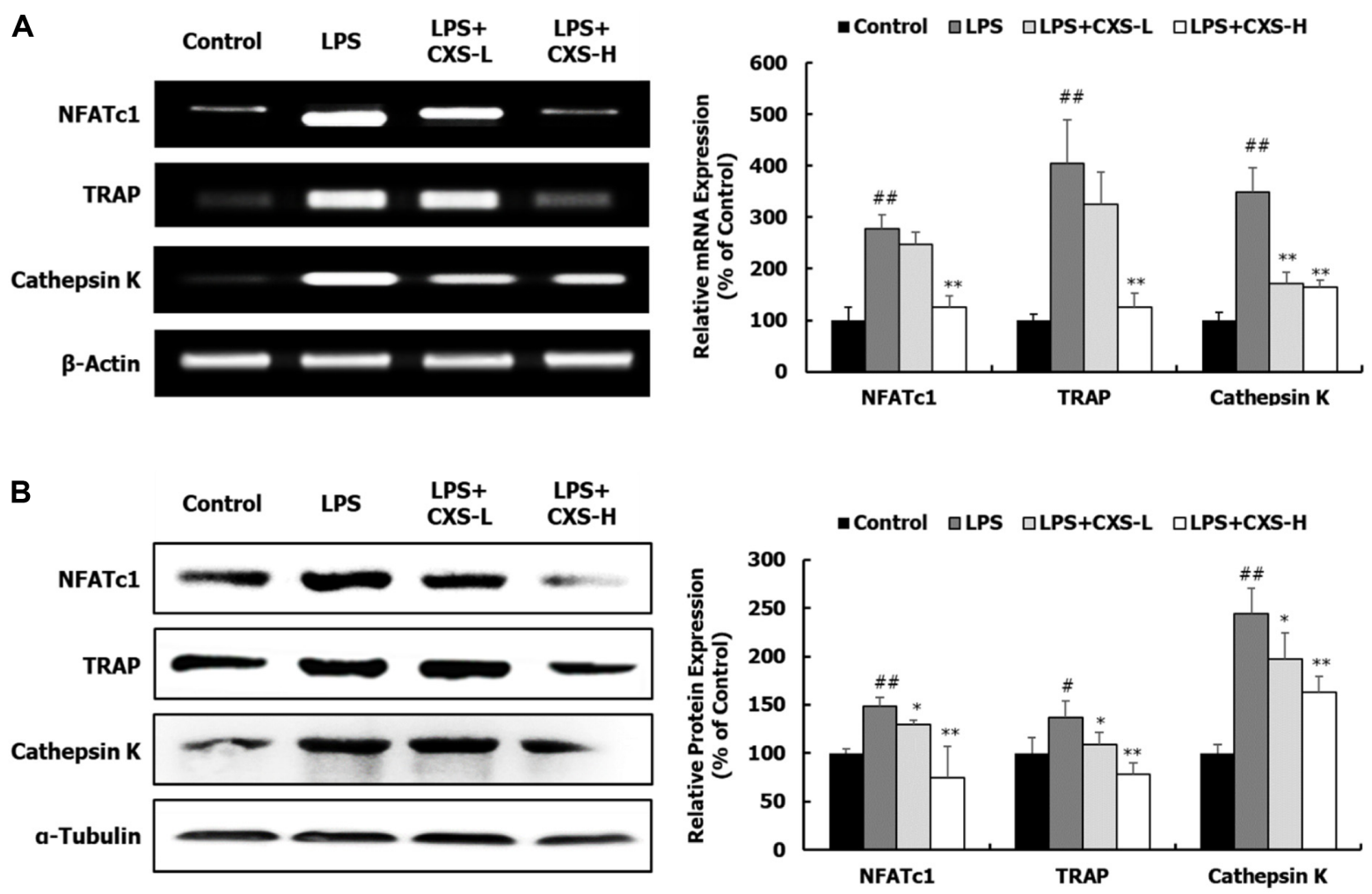

Fig. 2. Inhibitory effects of CXS on osteoclastic factors in alveolar bone of LPS-treated rats.

All the groups except control were injected with $1 \mathrm{mg} / \mathrm{ml}$ LPS every two days. Oral administration of CXS at the dose of $30 \mathrm{mg} \cdot \mathrm{kg}^{-1} \cdot \mathrm{day}^{-1}$ (LPS+CXS-L) and $100 \mathrm{mg} \cdot \mathrm{kg}^{-1} \cdot \mathrm{day}^{-1}$ (LPS+CXS-H) was started after six LPS injections and daily conducted for eight days. (A) mRNA levels of TRAP, cathepsin K, and NFATc1 were measured using RT-PCR with $\beta$-actin as the housekeeping gene. (B) Protein levels of TRAP, cathepsin K, and NFATc1 were measured by western blotting with $\alpha$-tubulin as the housekeeping gene. The relative expression levels were expressed as mean \pm standard deviation (SD, \% control) and group differences were assessed by Duncan's multiple range tests. ${ }^{\#} p<0.05$ and ${ }^{\# \#} p<0.01$ (Control group vs. LPS group); ${ }^{*} p<0.05$ and ${ }^{* *} p<0.01$ (LPS group vs. sample groups).

of p-p38, p-JNK, and p-ERK was suppressed (Fig. 3A). In particular, the expressions of p-p38, p-JNK, and p-ERK in the CXS-H group was decreased by $71.20 \%, 70.66 \%$, and $52.25 \%$, respectively, over the LPS group. The protein expression of AP-1 components, such as c-Fos and p-c-Jun, were downregulated in the CXS-H group (Fig. 3B). The expression of $\mathrm{p}$-c-Jun and c-Fos in the CXS-H group was decreased by $59.17 \%$ and $37.64 \%$, compared to the LPS group.

\section{CXS Improves Bone Homeostasis}

Since RANKL is an initiator of osteoclastogenesis and OPG is a decoy receptor of RANKL [23], stimulatory effect of CXS on OPG/RANKL ratio was analyzed. With 8 days of CXS administration, the mRNA and protein levels of OPG were significantly increased, but there was a marked decrease in the mRNA and protein expression of RANKL, compared to the level in the LPS group (Figs. 4A and 4B).
Consequently, the OPG/RANKL ratio at the mRNA and the protein levels was increased by $76.93 \%$ and $82.76 \%$, respectively, in the CXS-H group (Fig. 4). Since OPG is secreted from osteoblasts during osteoblastogenesis [24], the increased expression of OPG represents the occurrence of osteoblast differentiation. Therefore, this study was evaluated whether CXS had stimulatory effect on osteoblastogenesis. The mRNA and protein expression levels of ALP and COLA1 in the CXS-H group were higher than those in the LPS group (Figs. 5A and 5B). These results suggest that CXS improved bone homeostasis by inhibiting osteoclastogenesis and stimulating osteoblastogenesis in LPS-treated rats.

\section{CXS Improves Histological Changes}

The condition of periodontal ligaments was evaluated through H\&E staining to determine whether cellular infiltration occurred when periodontitis was induced in the 
A
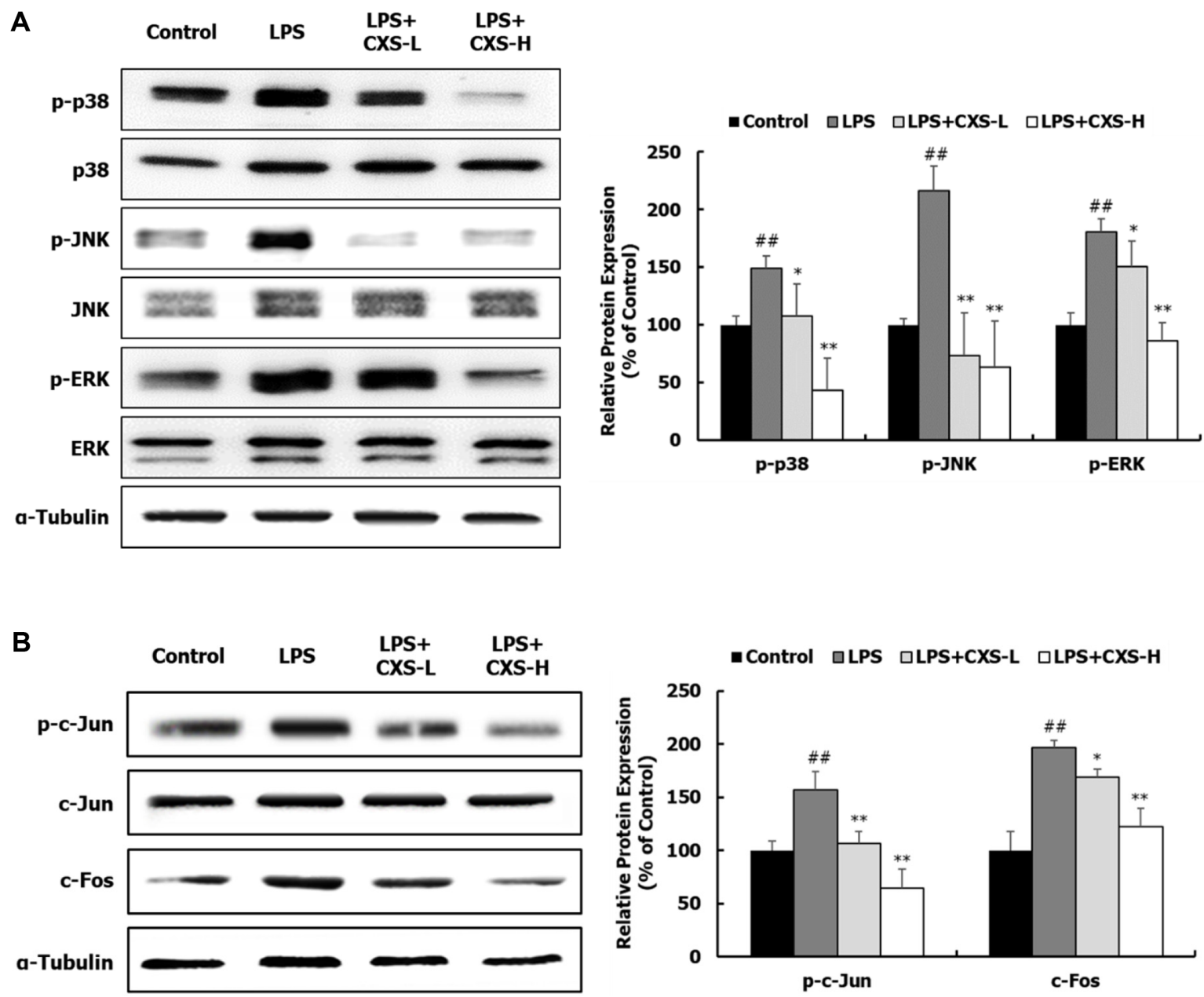

Fig. 3. Inhibitory Effects of CXS on MAPKs and AP-1 complex in alveolar bone of LPS-treated rats.

All the groups except control were injected with $1 \mathrm{mg} / \mathrm{ml}$ LPS every two days. Oral administration of CXS at the dose of $30 \mathrm{mg} \cdot \mathrm{kg}^{-1} \cdot \mathrm{day}^{-1}$ (LPS+CXS-L) and $100 \mathrm{mg} \cdot \mathrm{kg}^{-1} \cdot \mathrm{day}^{-1}$ (LPS+CXS-H) was started after six LPS injections and daily conducted for eight days. (A) Protein levels of p-JNK, p-ERK and p-p38 were detected by western blotting with specific antibodies. (B) Protein levels of p-c-Jun, c-Jun, and c-Fos were also detected by western blotting. $\alpha$-Tubulin was used as the housekeeping gene. The relative expression levels are expressed as mean \pm standard deviation (SD, \% control). Group differences were assessed by Duncan's multiple range tests. ${ }^{\#} p<0.01$ (Control group vs. LPS group); $p<0.05$ and ${ }^{* *} p<0.01$ (LPS group vs. sample groups).

animal model. It was observed that the LPS group had an uneven line of periodontal ligament between dentin and alveolar bone because of cellular infiltration, while the line in the CXS groups was recovered as the infiltration had been ameliorated (Fig. 6A). In addition, the expression of major periodontitis-related proteins was determined by immunohistochemistry (Fig. 6B). The protein expression of MMP-2, MMP-8, NFATc1, and TRAP was reduced in the CXS-H group, compared to those in the LPS group. The protein expression of ALP was dose-dependently upregulated by CXS, compared to that in the LPS group.
CXS Reduces the Distance of Alveolar Bone Loss by Improved Trabecular Spectrum

Alveolar bone loss in the animal model was measured through 2D micro-CT imaging of the linear length between CEJ and $\mathrm{ABC}$ (Fig. 7A). The distance of alveolar bone loss in the LPS group was $1.21 \pm 0.12 \mathrm{~mm}$, which was $44.67 \%$ longer than in the control group, while the distance in the CXS-L and the CXS-H groups was $4.92 \%$ and $24.51 \%$ shorter than that in the LPS group, respectively (Fig. 7B). In the 3D micro-CT imaging analysis, the numerical values of BV/TV and $\mathrm{Tb}$.Th. were regained in the CXS groups by as much as 
A
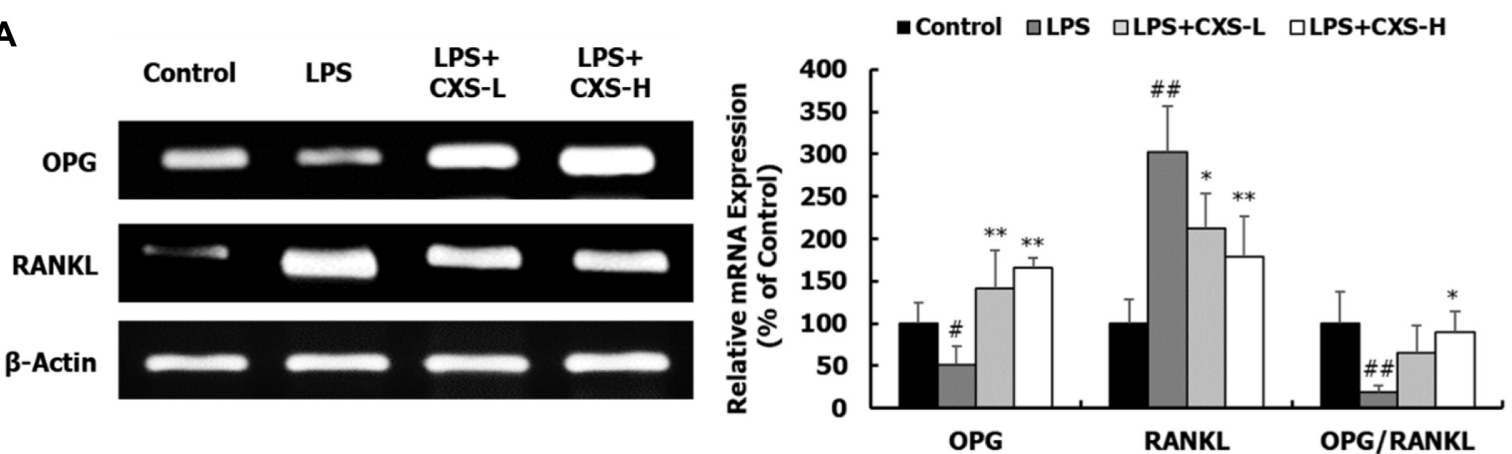

B
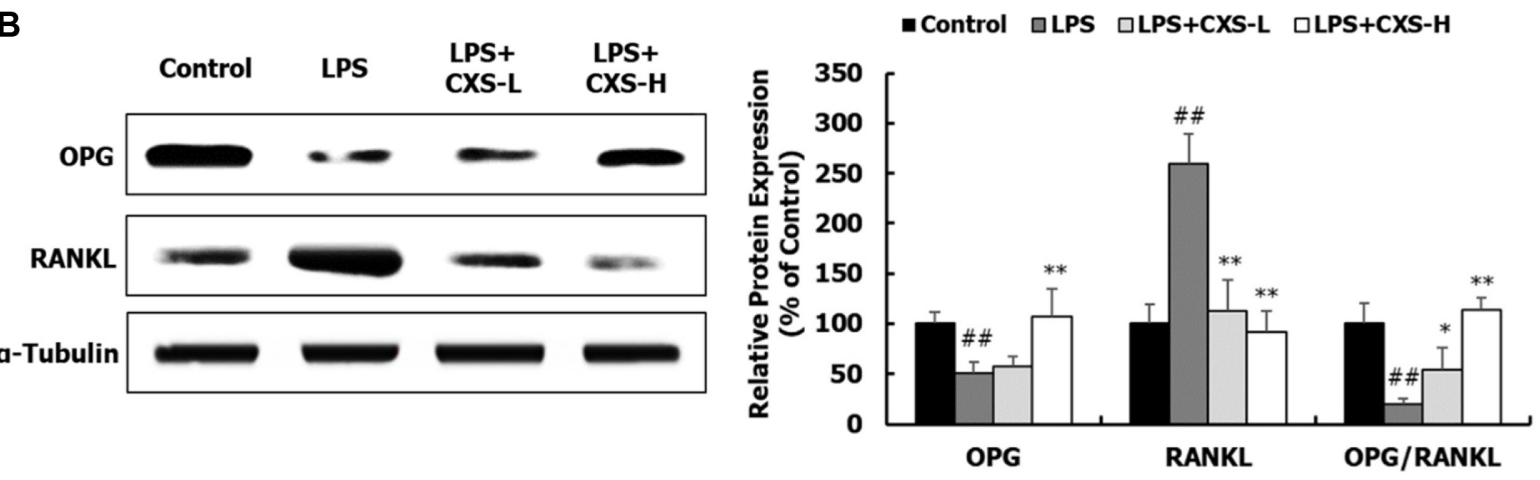

Fig. 4. Stimulatory effect of CXS on OPG/RANKL ratio in alveolar bone of LPS-treated rats.

All the groups except control were injected with $1 \mathrm{mg} / \mathrm{ml}$ LPS every two days. Oral administration of CXS at the dose of $30 \mathrm{mg} \cdot \mathrm{kg}^{-1} \cdot \mathrm{day}^{-1}$ (LPS+CXS-L) and $100 \mathrm{mg} \cdot \mathrm{kg}^{-1} \cdot \mathrm{day}^{-1}$ (LPS+CXS-H) was started after six LPS injections and daily conducted for eight days. (A) mRNA levels of OPG and RANKL were estimated using RT-PCR with $\beta$-actin as the housekeeping gene. (B) Protein levels of OPG and RANKL were measured by western blotting with $\alpha$-tubulin as the housekeeping gene. The relative expression levels are expressed as mean \pm standard deviation (SD, $\%$ control). Group differences were assessed by Duncan's multiple range tests. ${ }^{\# *} p<0.01$ (Control group vs. LPS group); ${ }^{*} p<0.05$ and ${ }^{* *} p<0.01$ (LPS group vs. sample groups).

the values in the control group (Figs. 7C and 7D). In contrast, the CXS groups showed a significant decline in Tb.Sp., which represents the spatial distance between trabecular bones (Fig. 7E). The value of BMD in the CXS-H group was $13.83 \%$ higher than that in the LPS group (Fig. 7F).

\section{Discussion}

Periodontitis is characterized by the inflammation of gingival tissue and alveolar bone destruction [9]. The present study evaluated whether CXS inhibited periodontal disease by attenuating periodontal inflammation and alveolar bone resorption. CXS downregulated major inflammatory factors, such as IL-1 $\beta$ and NF- $\mathrm{BB}$ in periodontal tissue (Fig. 1); this anti-inflammatory effect might be mediated by xanthorrhizol and other phytochemicals in C. xanthorrhiza, such as $\alpha$-pinene, camphor, and curcumin [25-27]. In particular, C. xanthorrhiza has a high content of xanthorrhizol, which reportedly possesses anti-inflammatory effect in LPS-treated RAW 264.7 [28]. Therefore, the mixture of xanthorrhizol and phytochemicals in CXS might attenuate periodontitis by inhibiting the inflammation.

In the series of the signaling pathway, AP-1 complex stimulates the expression of IL- $1 \beta, N F-\kappa B$, and the periodontal ligament degradation-related enzymes, such as MMP-2 and MMP-8 [29]. MMPs degrade proteins, such as collagen, gelatin, and elastin in the extracellular matrix. Therefore, the overexpression of MMPs causes the destruction of gingival fibers. In this study, MMP-2 and MMP-8 were upregulated in the LPS group and CXS downregulated the expression of MMPs (Fig. 1). Furthermore, the ameliorative effect of CXS on periodontitis was supported by verifying the histological and phenomenological changes in periodontal tissues. Periodontal inflammation in the periodontal ligament is followed by the overexpression of immune cells, such as macrophages and lymphocytes, which accelerate 
A
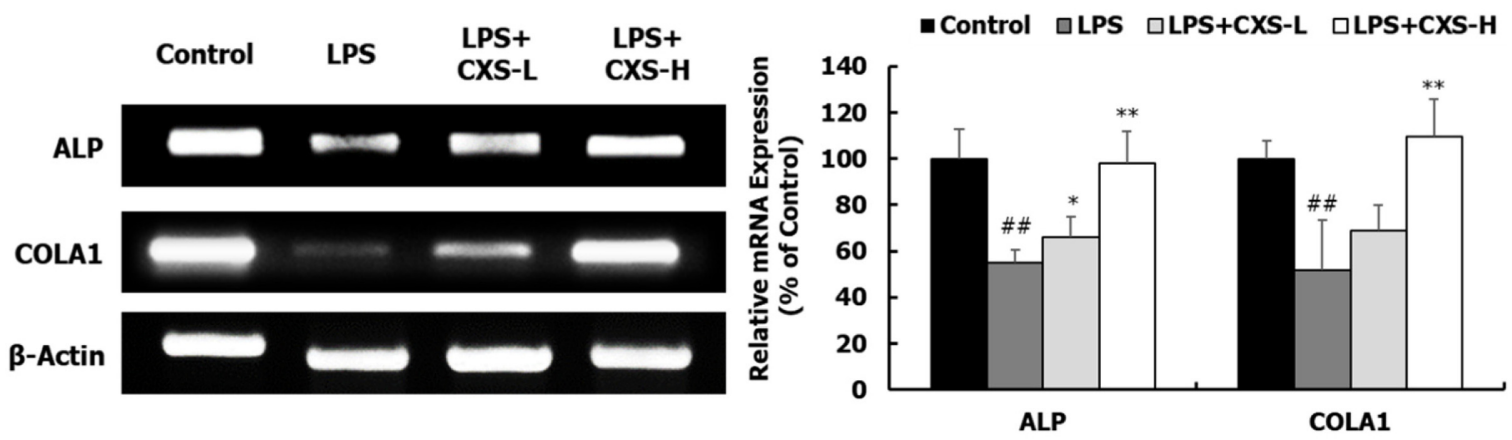

B
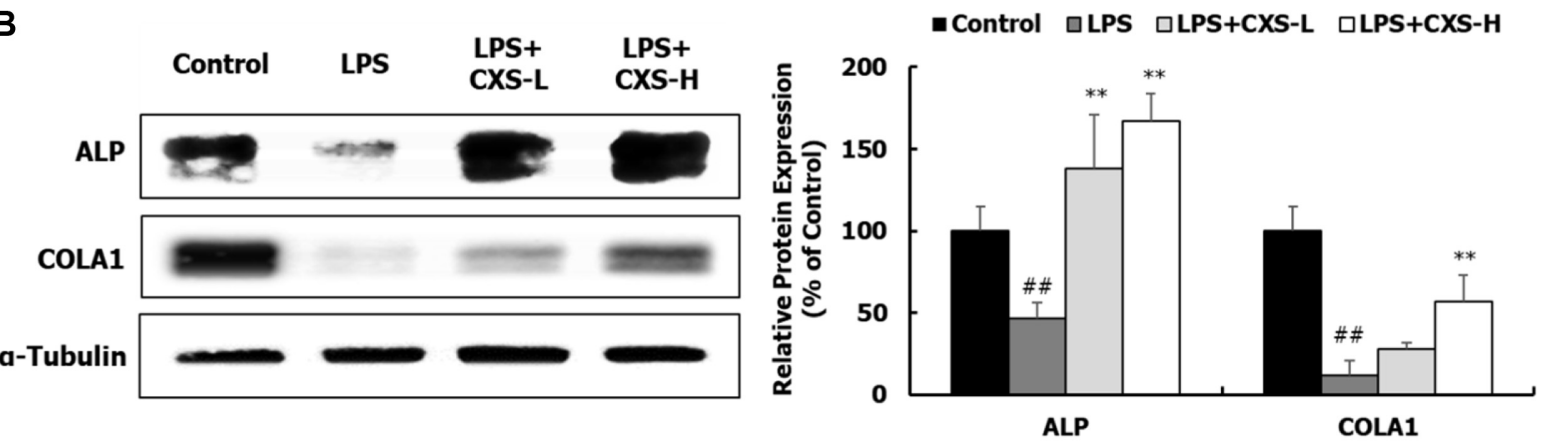

Fig. 5. Stimulatory effects of CXS on osteogenesis-related markers in alveolar bone of LPS-treated rats.

All the groups except control were injected with $1 \mathrm{mg} / \mathrm{ml}$ LPS every two days. Oral administration of CXS at the dose of $30 \mathrm{mg} \cdot \mathrm{kg}^{-1} \cdot \mathrm{day}^{-1}$ (LPS+CXS-L) and $100 \mathrm{mg} \cdot \mathrm{kg}^{-1} \cdot \mathrm{day}^{-1}$ (LPS+CXS-H) was started after six LPS injections and daily conducted for eight days. (A) mRNA levels of ALP and COLA1 were estimated using RT-PCR with $\beta$-actin as the housekeeping gene. (B) Protein levels of ALP and COLA1 were measured by western blotting with $\alpha$-tubulin as the housekeeping gene; the relative expression levels are expressed as mean \pm standard deviation (SD, \% control). Group differences were assessed by Duncan's multiple range tests. ${ }^{\# *} p<0.01$ (Control group vs. LPS group); ${ }^{*} p<0.05$ and ${ }^{* *} p<0.01$ (LPS group vs. sample groups).

cell infiltration. CXS attenuated the cell infiltration on the boundary line of the periodontal ligament (Fig. 6A). Therefore, CXS had an inhibitory effect on inflammation by attenuating the overactivation of immune cells in the periodontal ligament.

Persistent inflammation on the periodontium accelerates osteoclastogenesis in which the upregulation of NFATc1 as a transcription factor stimulates the major osteoclastic factors, such as TRAP and cathepsin K [22, 30]. The osteoclastic factors were overexpressed in the LPS group, while CXS diminished the expressions of TRAP and cathepsin K (Fig. 2). In this study, CXS inactivated MAPKs and AP-1 complex in alveolar bone (Fig. 3). Since the MAPKs signaling pathway contributes to occurrence of alveolar bone loss by stimulating the heterodimerization of AP-1 components, such as c-Jun and c-Fos [30, 31], CXS inhibited osteoclastogenesis by blocking MAPK/AP-1 signaling pathways. In bone homeostasis, OPG, which is a decoy receptor of the TNF receptor family, is secreted during osteoblast differentiation [32]. Secreted OPG binding to RANKL inhibits osteoclastogenesis by preventing RANKLRANK binding, consequently, reducing osteoclastogenesis and bone resorption [23]. CXS increased OPG expression while decreasing RANKL expression (Fig. 4). In addition, the ratio between OPG/RANKL was increased by CXS. It could be explained that CXS contributed to the inhibition of osteoclastogenesis by blocking RANKL-RANK binding and to the remodeling of alveolar bone.

CXS could be considered to have osteoblastogenic effect. Osteoblastogenesis is regulated by two major proteins, ALP and COLA1 [33]. ALP is the significant biological marker for proliferation during the differentiation of osteoblasts, and mainly regulates bone crystallization [34]. COLA1 is a skeletal matrix protein that promotes osteoblast differentiation and maintains bone quality [15]. In this study, the expression of ALP and COLA1 was examined at 
A

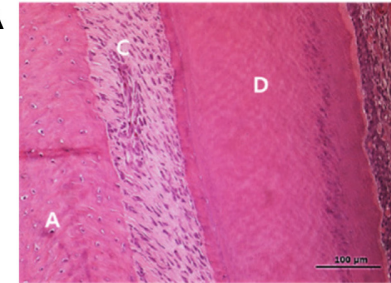

Control

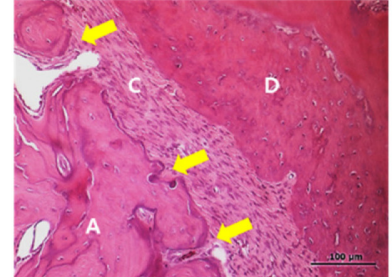

LPS

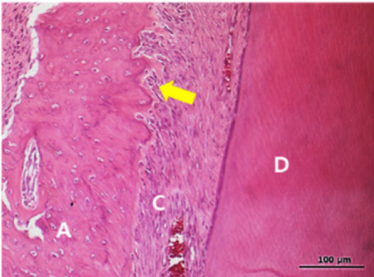

LPS+CXS-L

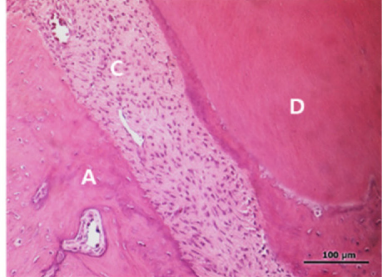

LPS+CXS-H

B

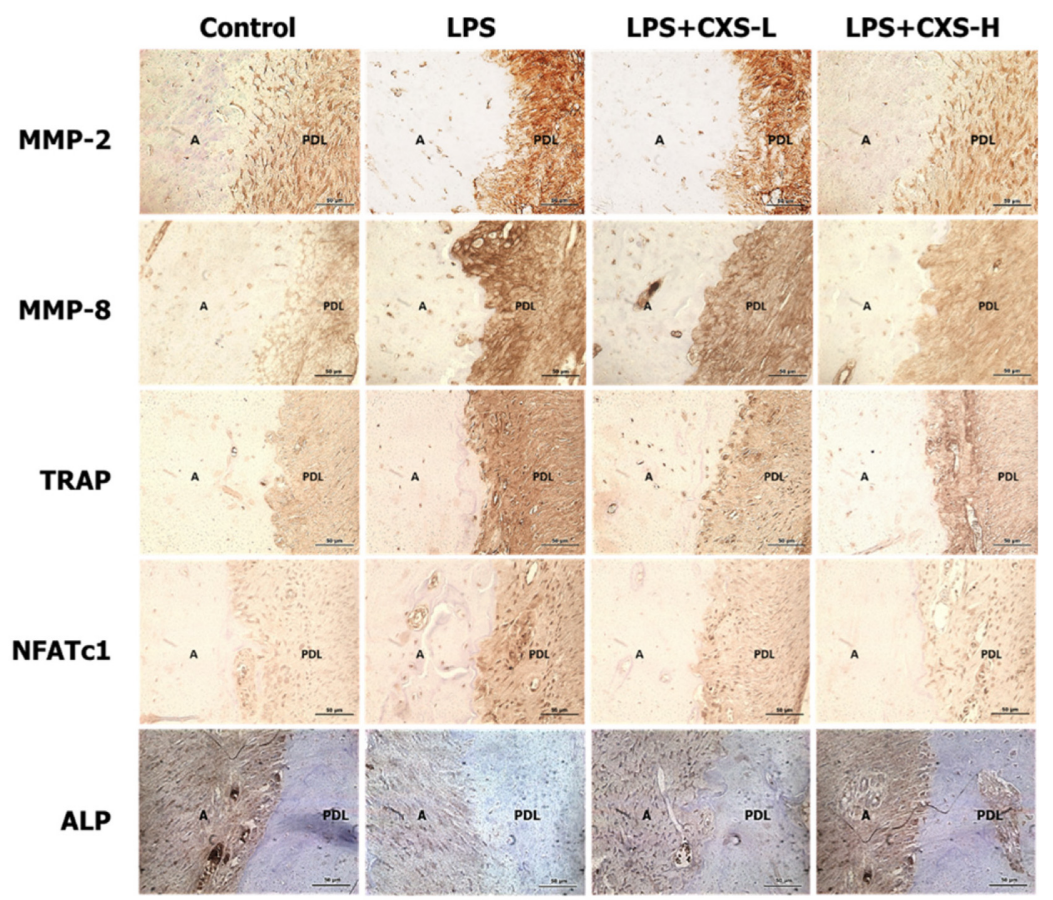

C

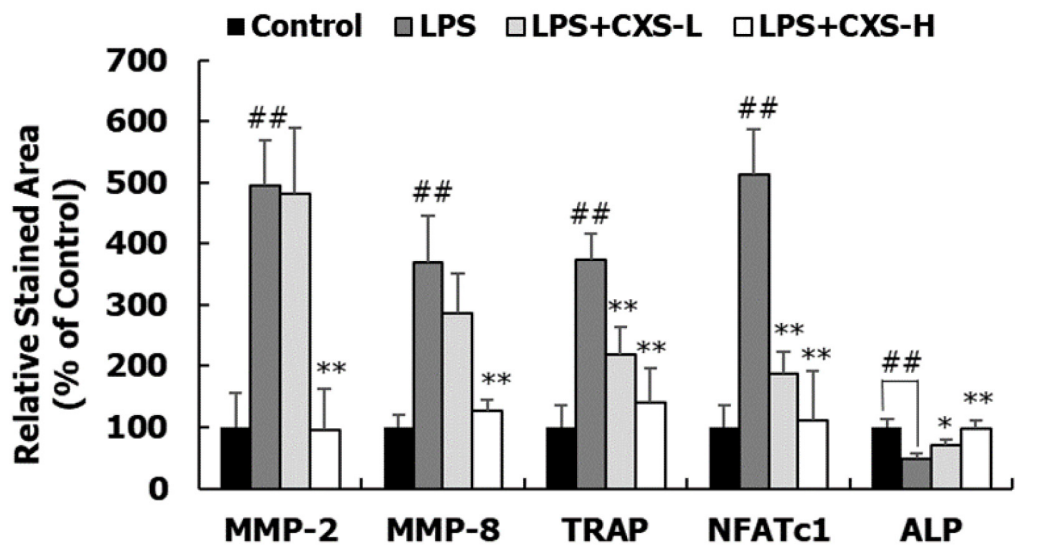

Fig. 6. Effects of CXS on histological changes in LPS-treated rats.

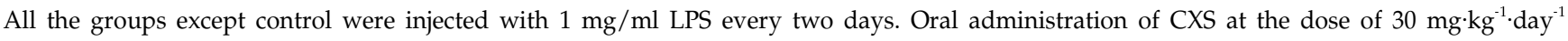
(LPS+CXS-L) and $100 \mathrm{mg} \cdot \mathrm{kg}^{-1} \cdot \mathrm{day}^{-1}$ (LPS+CXS-H) was started after six LPS injections and daily conducted for eight days. (A) Histological analysis of periodontium using H\&E staining (magnification, $\times 200$ ). Yellow arrow, cell infiltration; A, alveolar bone; C, connective tissue; D, dentin. (B) Histological analysis of periodontium using immunohistochemistry (magnification, $\times 400$ ). A, alveolar bone; PDL, periodontal ligament. (C) Quantification of relative stained areas in immunohistochemistry images. The relative stained areas are expressed as mean \pm standard deviation (SD, \% control). Group differences were assessed by Duncan's multiple range tests. ${ }^{* \#} p<0.01$ (Control group vs. LPS group); ${ }^{*} p<0.05$ and ${ }^{* *} p<$ 0.01 (LPS group vs. sample groups). 
A

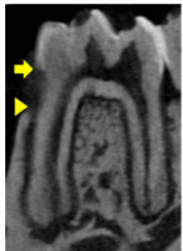

Control

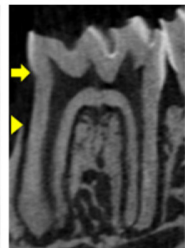

LPS

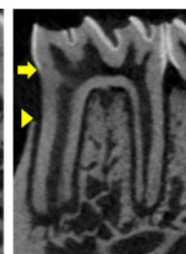

LPS+CXS-L

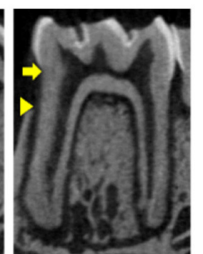

LPS+CXS-H

\section{B}

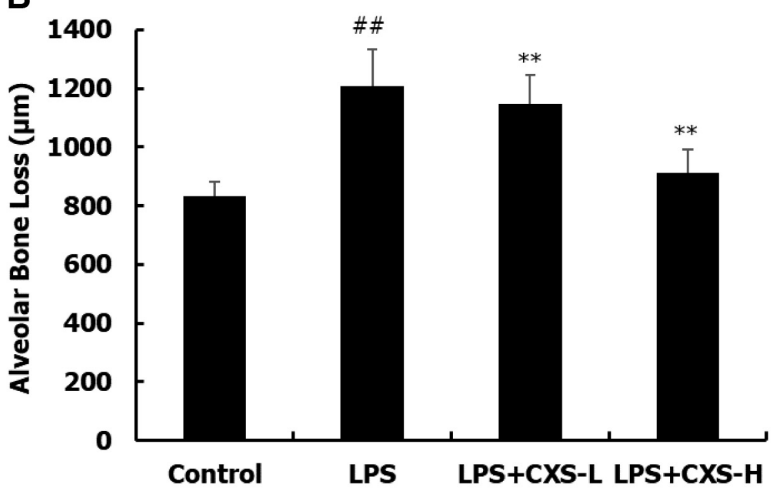

C

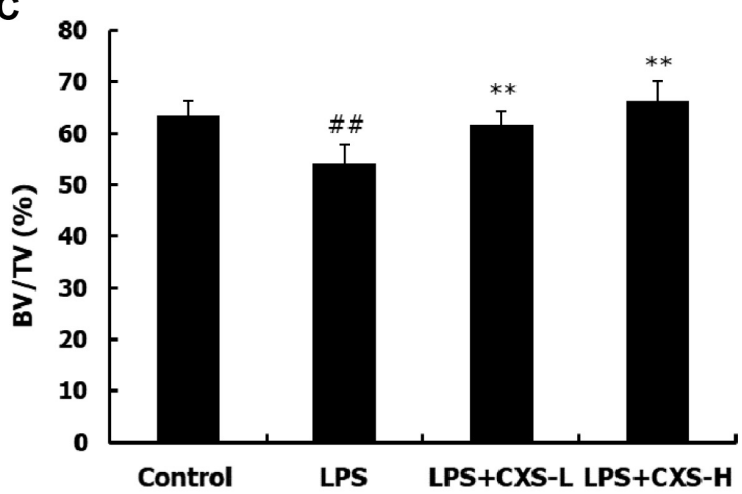

。

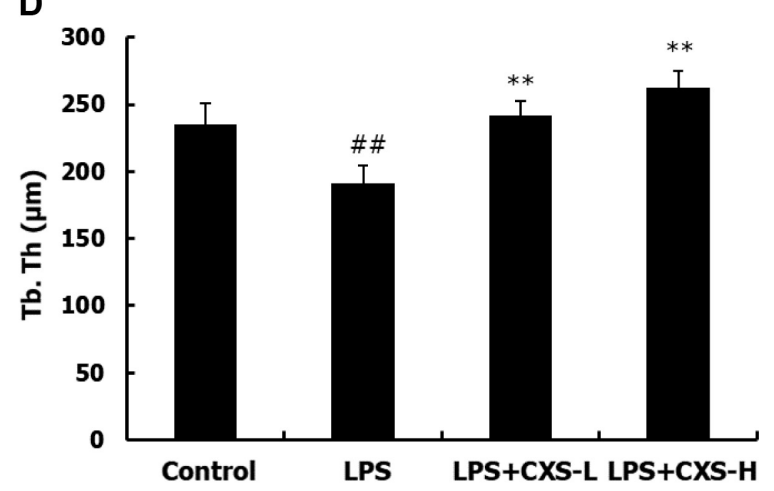

$\mathbf{F}$

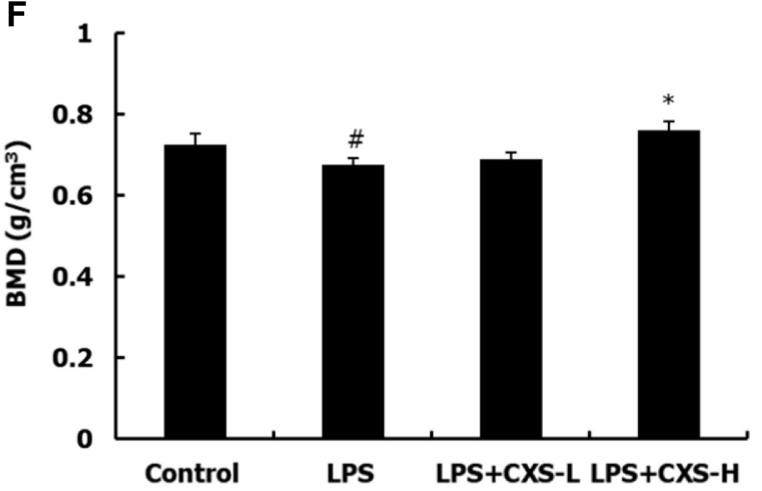

Fig. 7. Ameliorative effect of CXS on alveolar bone loss and bone destruction in LPS-treated rats.

All the groups except control were injected with $1 \mathrm{mg} / \mathrm{ml}$ LPS every two days. Oral administration of CXS at the dose of $30 \mathrm{mg} \cdot \mathrm{kg}^{-1} \cdot \mathrm{day}^{-1}$ (LPS+CXS-L) and $100 \mathrm{mg} \cdot \mathrm{kg}^{-1} \cdot \mathrm{day}^{-1}$ (LPS+CXS-H) was started after six LPS injections and daily conducted for eight days. (A) Micro-CT 2D images and (B) quantification of the CEJ-ABC distance. Arrow, CEJ; triangle, ABC. (C) BV/TV, (D) Tb. Th., and (E) Tb. Sp., and (F) bone mineral density (BMD) of alveolar bone covering the root of the first molar were measured using reconstructed 3D images. The alveolar bone loss and each parameter are expressed as mean \pm standard deviation (SD, $\%$ or $\mu \mathrm{m}$ ). Group differences were assessed by Duncan's multiple range tests. ${ }^{*} p<0.05$ and ${ }^{\# \#} p<0.01$ (Control group vs. LPS group); ${ }^{*} p<0.05$ and ${ }^{* *} p<0.01$ (LPS group vs. sample groups.

the molecular level (Fig. 5). The protein expression of ALP in the CXS-H group was $259.56 \%$ higher than that in the LPS group. This value was also $40.10 \%$ higher, compared to that in the control group. These results represent that CXS has a stimulatory effect on osteoblastogenesis.

Since CXS had a stimulatory effect on osteoblastogenesis and inhibitory effect of osteoclastogenesis, the first mandible tooth was used to evaluate the degree of alveolar bone 
formation through micro-CT. Bone trabecular parameters are determined to explain density of bone meshwork [35]. $\mathrm{BV} / \mathrm{TV}$ indicates the occupied region of bone among the whole region of tissues. Trabecular parameters represent mandible bone morphology; $\mathrm{Tb}$.Th. indicates the thickness of the trabecula, while Tb.Sp. implies the separation distance between trabecular bones [36]. The CXS-H group revealed the decline of distance between $\mathrm{CEJ}$ and $\mathrm{ABC}$ (Figs. 7A and 7B). In addition, the value of $\mathrm{Tb}$.Th was increased and that of $\mathrm{Tb} . \mathrm{Sp}$ was decreased in the CXS groups (Figs. 7D and 7E). These results indicate that the bone loss is alleviated and bone formation occurs by CXS treatment.

The present study demonstrated whether CXS attenuated periodontal inflammation and alveolar bone resorption on LPS-induced periodontitis in an animal model. CXS significantly reduced the expression of periodontal inflammation-related and osteoclastogenesis-related biomarkers and increased osteoblast differentiation-related factors in LPS-treated rats; thus, CXS had three major effects on periodontal tissue: the ability to suppress gingival inflammation, to inhibit alveolar bone loss, and to enhance bone formation. These pre-clinical results recommend further studies in humans for the anti-periodontitis effect of CXS. Collectively, CXS could be used as a natural antiperiodontitis agent in the amelioration of periodontal disease.

\section{Acknowledgments}

This research has been funded in part by the 2016 Yonsei University Future-leading Research Initiative (RMS2 201611-1540) and the World Class 300 Project R\&D Program (S2435140), supported by the Small and Medium Business Administration (SMBA, Republic of Korea).

\section{Conflict of Interest}

The authors have no financial conflicts of interest to declare.

\section{References}

1. Cekici A, Kantarci A, Hasturk H, Van Dyke TE. 2014. Inflammatory and immune pathways in the pathogenesis of periodontal disease. Periodontol. 2000 64: 57-80.

2. Singh N, Chandel S, Singh H, Agrawal A, Savitha A. 2017. Effect of scaling \& root planing on the activity of ALP in GCF \& serum of patients with gingivitis, chronic and aggressive periodontitis: a comparative study. J. Oral Biol.
Craniofac. Res. 2017: 123-126.

3. Hajishengallis G. 2015. Periodontitis: from microbial immune subversion to systemic inflammation. Nat. Rev. Immunol. 15: 30-44.

4. Barton MK. 2017. Evidence accumulates indicating periodontal disease as a risk factor for colorectal cancer or lymphoma. CA Cancer J. Clin. 67: 173-174.

5. Merchant AT, Virani SS. 2017. Evaluating periodontal treatment to prevent Cardiovascular disease: challenges and possible solutions. Curr. Atheroscler. Rep. 19: 4-9.

6. Harding A, Robinson S, Crean SJ, Singhrao SK. 2017. Can better management of periodontal disease delay the onset and progression of Alzheimer's disease? J. Alzheimers Dis. 2017: 337-348.

7. Hasan A, Palmer RM. 2014. A clinical guide to periodontology: pathology of periodontal disease. Br. Dent. J. 216: 457-461.

8. Chiu HC, Fu MM, Yang TS, Fu E, Chiang CY, Tu HP, et al. 2017. Effect of high glucose, Porphyromonas gingivalis lipopolysaccharide and advanced glycation end-products on production of interleukin-6/-8 by gingival fibroblasts $J$. Periodontal. Res. 52: 268-276.

9. Hienz SA, Paliwal S, Ivanovski S. 2015. Mechanisms of bone resorption in periodontitis. J. Immunol. Res. 2015: 615486.

10. Müller HD, Cvikl BB, Lussi AA, Gruber RR. 2016. Salivary pellets induce a pro-inflammatory response involving the TLR4-NF-kB pathway in gingival fibroblasts. BMC Oral Health. 17: 15-24.

11. Franco C, Patricia H-R, Timo S, Claudia B, Marcela H. 2017. Matrix metalloproteinases as regulators of periodontal inflammation. Int. J. Mol. Sci. 18: 440-451.

12. Maeso G, Bravo M, Bascones A. 2007. Levels of metalloproteinase-2 and-9 and tissue inhibitor of matrix metalloproteinase-1 in gingival crevicular fluid of patients with periodontitis, gingivitis, and healthy gingiva. Quintessence Int. 38: 247-252.

13. Scannapieco FA. 2004. Periodontal inflammation: from gingivitis to systemic disease? Compend. Contin. Educ. Dent. 25: $16-25$.

14. He X, Andersson G, Lindgren U, Li Y. 2010. Resveratrol prevents RANKL-induced osteoclast differentiation of murine osteoclast progenitor RAW 264.7 cells through inhibition of ROS production. Biophys. Res. Commun. 401: 356-362.

15. Algate K, Haynes DR, Bartold PM, Crotti TN, Cantley MD. 2016. The effects of tumour necrosis factor- $\alpha$ on bone cells involved in periodontal alveolar bone loss; osteoclasts, osteoblasts and osteocytes. J. Periodontal Res. 51: 549-566.

16. Kim JE, Kim HE, Hwang JK, Lee HJ, Kwon HK, Kim BI. 2008. Antibacterial characteristics of Curcuma xanthorrhiza extract on Streptococcus mutans biofilm. Int. J. Microbiol. 46: 228-232.

17. Mary HP, Susheela GK, Jayasree S, Nizzy A, Rajagopal B, Jeeva S. 2012. Phytochemical characterization and antimicrobial activity of Curcuma xanthorrhiza Roxb. Asian Pac. J. Trop. 
Biomed. 2: S637-S640.

18. Kim B, Kim S, Chang S, Moon J, Kim Y, Hwang JK, et al. 2005. A highly selective antibacterial effect of Curcuma xanthorrhiza extract against oral pathogens and clinical effectiveness of a dentifrice containing Curcuma xanthorrhiza extract for controlling bad breath. J. Korean Acad. Oral Health. 29: 222-237.

19. Cho JY, Kim HY, Kim HM, Song HN, Hong E, Hwang JK, et al. 2017. Standardized ethanolic extract of the rhizome of Curcuma xanthorrhiza prevents murine ulcerative colitis by regulation of inflammation. J. Funct. Foods 30: 282-289.

20. Ab Halim MR, Tan M, Ismail S, Mahmud R. 2012. Standardization and phytochemical studies of Curcuma xanthorrhiza Roxb. Int. J. Pharm. Pharm. Sci. 4: 606-610.

21. Oon SF, Nallappan M, Tee TT, Shohaimi S, Kassim NK, Sa'ariwijaya MSF, et al. 2015. Xanthorrhizol: a review of its pharmacological activities and anticancer properties. Cancer Cell Int. 15: 100-114.

22. Seo T, Cha S, Kim TI, Lee JS, Woo KM. 2012. Porphyromonas gingivalis-derived lipopolysaccharide-mediated activation of MAPK signaling regulates inflammatory response and differentiation in human periodontal ligament fibroblasts. J. Microbiol. 50: 311-319.

23. Walsh MC, Choi Y. 2014. Biology of the RANKL-RANKOPG system in immunity, bone, and beyond. Front. Immunol. 2014: 511-521.

24. Xu T, Wang L, Tao Y, Ji Y, Deng F, Wu XH. 2016. The function of naringin in inducing secretion of osteoprotegerin and inhibiting formation of osteoclasts. Evid. Based Complement. Alternat. Med. 2016: 8981650.

25. Ehrnhöfer-Ressler MM, Fricke K, Pignitter M, Walker JM, Walker J, Rychlik M, et al. 2013. Identification of 1, 8cineole, borneol, camphor, and thujone as anti-inflammatory compounds in a Salvia officinalis L. infusion using human gingival fibroblasts. J. Agric. Food Chem. 61: 3451-3459.

26. Hu P, Huang P, Chen MW. 2013. Curcumin attenuates cyclooxygenase-2 expression via inhibition of the NF- $\mathrm{KB}$ pathway in lipopolysaccharide-stimulated human gingival fibroblasts. Cell Biol. Int. 37: 443-448.

27. Rufino AT, Ribeiro M, Judas F, Salgueiro L, Lopes MC, Cavaleiro C, et al. 2014. Anti-inflammatory and chondroprotective activity of (+)- $\alpha$-pinene: structural and enantiomeric selectivity. J. Nat. Prod. 77: 264-269.

28. Lim CS, Jin DQ, Mok H, Oh SJ, Lee JU, Hwang JK, et al. 2005. Antioxidant and antiinflammatory activities of xanthorrhizol in hippocampal neurons and primary cultured microglia. J. Neurosci. Res. 82: 831-838.

29. Ko SY. 2016. Inhibitory effect of myricetin on matrix metalloproteinase expression and activity in periodontal inflammation. Int. J. Oral Biol. 41: 163-173.

30. Lee K, Chung YH, Ahn H, Kim H, Rho J, Jeong D. 2016. Selective regulation of MAPK signaling media ANKLtes R-dependent osteoclast differentiation. Int. J. Biol. Sci. 12: 235-245.

31. Li Q, Valerio MS, Kirkwood KL. 2012. MAPK usage in periodontal disease progression. J. Signal Transduct. 2012: 308943.

32. Kostenuik PJ. 2005. Osteoprotegerin and RANKL regulate bone resorption, density, geometry and strength. Curr. Opin. Pharmacol. 5: 618-625.

33. Ram VS, Parthiban, Sudhakar U, Mithradas N, Prabhakar R. 2015. Bonebiomarkers in periodontal disease: a review article. J. Ciln. Diagn. Res. 9: ZE07-ZE10.

34. Hessle L, Johnson KA, Anderson HC, Narisawa S, Sali A, Goding JW, et al. 2002. Tissue-nonspecific alkaline phosphatase and plasma cell membrane glycoprotein-1 are central antagonistic regulators of bone mineralization. Proc. Natl. Acad. Sci. USA 99: 9445-9449.

35. Buie HR, Campbell GM, Klinck RJ, MacNeil JA, Boyd SK. 2007. Automatic segmentation of cortical and trabecular compartments based on a dual threshold technique for in vivo micro-CT bone analysis. Bone 41: 505-515.

36. Swain MV, Xue J. 2009. State of the art of Micro-CT applications in dental research. Int. J. Oral Sci. 1: 177-188. 